\title{
Stability Analysis of Centerless Grinding with Loss of Contact Nonlinearity
}

\author{
Marco Leonesio ${ }^{1}$, Giacomo Bianchi ${ }^{1}$ and Hossein Safarzadeh ${ }^{2}$ \\ ${ }^{1}$ CNR - STIIMA, Institute of Intelligent Industrial Technologies and Systems for Advanced Manufacturing - National Research Council \\ of Italy, IT-20133 Milan, Italy \\ ${ }^{2}$ Politecnico diMilano, Dipartimento di Meccanica, IT-20156 Milano, Italy
}

\begin{abstract}
The paper presents a novel geometrical stability analysis of centerless grinding that takes into account the nonlinearity associated to wheel-workpiece detachment during lobes formation. Even though the rounding mechanism in centerless grinding has been studied since more than fifty years, stability analysis has been carried out applying stability criteria for linear systems (e.g., Nyquist) on a process model that neglects actual removal "clipping" due to wheel-workpiece detachment. This model limitation is usually overcome by considering only an integer number of lobes, supporting the restriction by the claim that a non-integral number of waves is less likely to build up since the waviness must be constantly removed and replaced by a succeeding wave, which is constantly moving around the workpiece. In this work, the nonlinearity entailed by removal clipping is explicitly taken into account and, by harmonic linearization, represented by a double input describing function (DIDF). Applying the Nyquist criterion on the resulting equivalent delayed system, the paramount instability associated to a quasi-integer number of lobes emerges naturally, without requiring additional assumptions. Moreover, it is shown that the nonlinearity due to wheel-workpiece detachment does not produce a limit cycle in a reasonable operation time. The results delivered by the proposed approach are verified by numeric simulations and positively compared to the relevant literature. The proposed formulation can be easily extended to consider also machine structure dynamics, thus increasing, even in this case, the accuracy of the stability analysis provided by the standard approach.
\end{abstract}

\section{Introduction}

Centerless grinding is a broadly used manufacturing process because of its unique workpiece (WP) holding system which leads to high-productivity and high accuracy. In this type of machine, the WP is supported on three points by the grinding wheel, the control wheel and the supporting blade (see Fig. 1), eliminating the need for clamping and centering holes on WP. WP loading and unloading is therefore easier, reducing the cycle time. But, on the other hand, due to this configuration, centerless grinding is subjected to roundness errors produced by two types of instabilities: regenerative chatter and geometric lobing ${ }^{1}$.

Chatter appears because of the interaction between the cutting process and the main resonances of the machine structure. Geometric lobing, instead, depends only on the geometric setup of the WP, i.e., blade angle and WP height, and it occurs even in case of perfectly rigid systems. It represents one of the main restrictions to WP roundness accuracy ${ }^{3}$ : WP center can oscillate and produce a polyhedral profile $\mathrm{e}^{4}$. Although the real machine and grinding wheels (GWs) are not perfectly rigid and system compliance reduces actual material removal, the focus of this study, as typically done in the literature, is to demonstrate how interrupted cut can produce geometric instability at integer lobe numbers even in a pure kinematic model. Additional flexibility effects, due to machine and spindle compliance, will be covered in a separated study.

In 1946, Dall carried on the first important study on the WP out-of-roundness problem, in which the roundness error was methodically related to the geometric configuration considering two basic parameters, the tangent angle and the top supporting blade assuming a perfectly rigid machine ${ }^{5}$. Yonetsu defined relationships between pre and post grinding amplitudes of harmonics of the WP profile ${ }^{6}$. In 1960s Rowe simulated the centerless grinding lobing problem on a digital computer taking into account all the previous geometrical considerations ${ }^{7-9}$ and presented the analytical model of the so-called geometric rounding mechanism. Later, these results were used to define the geometric roundness error regeneration process ${ }^{10}$. After defining the basic geometrical relationships of the process, it was possible to use different stability criteria, like Nyquist criterion, to show the theoretical instabilities produced by different configurations. Various researchers claimed that it is possible to create a stability map using the Nyquist criterion for each 
possible number of complete undulations that can be generated on the $\mathrm{WP}^{11-14}$. Zhou ${ }^{1}$ presented the periodic characteristic roots distribution of the lobing loop and proposed a nominal stability diagram to facilitate the selection of the center-height angle in order to minimize the lobing effect. Rowe ${ }^{15,16}$ presented the geometric stability parameter derived from the Nyquist stability criterion, restricted to integer lobes. Gallego ${ }^{17}$, Lizarralde $^{18}$, Hashimoto $^{19}$ and Madariaga ${ }^{20}$ have applied similar approaches to guide set up and optimization of centerless plunge grinding processes, in order to reduce set-up time and avoid geometric instabilities as a function of WP height and blade angle, taking into account machine-WP dynamic interaction. These techniques lead to models that quantitatively predict the evolution of profile error for each geometric configuration $^{18}$.

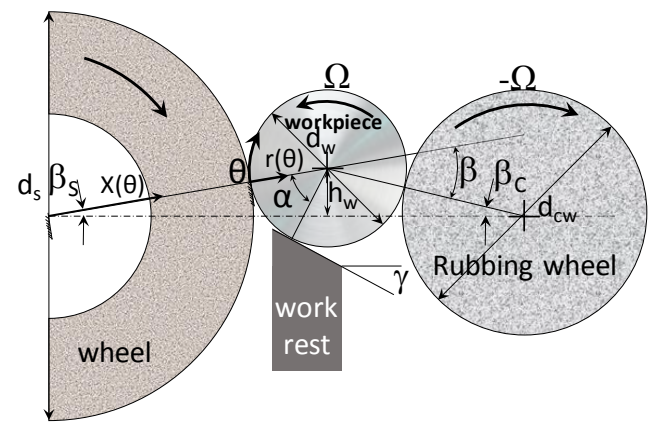

Fig. 1. Centerless grinding geometry

Even though the rounding mechanism in centerless grinding has been studied since more than fifty years, stability analysis has been carried out applying stability criteria for linear systems (e.g., Nyquist) on a process model that neglects actual removal "clipping" due to wheel-WP detachment (also denoted as negative depth of cut). Clipping has been considered in numerical simulation models ${ }^{8}$, but, as documented above, never in analytical analyses. When dealing with the pure geometrical mechanism - namely, without considering the one-per-turn delay due to work-wheel relative compliance - this model limitation yields wrong results, predicting the formation of a non-integer number of lobes, contradicting empirical evidence. Such discrepancy is usually overcome considering only integer number of lobes, supporting the restriction by the claim that "a non-integral number of waves is less likely to build up since the waviness must be constantly removed and replaced by a succeeding wave, which is constantly moving around the WP"21.

In this work we demonstrate that, for a pure geometrical instability, the formation of an integer number of lobes is due to the clipping nonlinearity. In our model, this nonlinearity is explicitly taken into account by harmonic linearization and represented by a Double Input Describing Function (DIDF). Applying the Nyquist criterion on the resulting equivalent delayed system, the paramount instability associated to a quasiinteger number of lobes (a slight lobes precession is often present) emerges naturally, without requiring any additional assumption. We show that the DIDF modifies the system introducing a delay loop corresponding to one WP revolution. This loop is formally similar to the one obtained when a wheel-workpiece compliance is considered (indicated as regenerative cutting action ${ }^{1}$ ), which also produces an integer number of lobes. Finally, we show that steady state waviness could entail the presence of more than one harmonic, thus violating the assumptions for DIDF validity: even though this situation has a limited practical interest, it could be treated with other techniques in future works.

The paper is structured as follows. In Section 2, the process model is presented: it includes the rounding mechanism, contact filtering and the clipping nonlinearity modelled by the DIDF. In Section 3, the process model is analyzed through Nyquist stability criterion. In Section 4, numerical process simulations are executed and compared to the analytical predictions by time frequency analysis. Discussion and conclusions are reported in Section 5.

\section{Process model}

\subsection{Geometric rounding mechanism}

Let a plunge centerless grinding be considered, described in Fig. 1. The shape of the cylindrical WP is defined by the actual radius reduction at the grinding point (i.e. at the contact between wheel and WP) ${ }^{21}$. The radius reduction is denoted with $r(\theta)$, where $\theta$ defines the WP rotation and starts from zero at the beginning of grinding. The reduction $r(\theta)$ cumulates during grinding and it is equal to the reduction at the previous revolution $r(\theta-2 \pi)$ summed to the current wheel-WP depth of cut $I(\theta)$ (also known as intersection, or engagement). Namely:

$$
r(\theta)=I(\theta)+r(\theta-2 \pi)
$$

Assuming a perfectly rigid system, the depth of cut is obtained from a pure kinematic model, computing the intersection between the grinding wheel and the WP, taking into account the feed movement $X(\theta)$, projected along the WP radius at grinding point, and WP center displacement due to WP profile contacts at work rest and rubbing wheel ${ }^{2}$.

Given its physical meaning, $I(\theta)$ cannot become negative, as the process either subtracts material, or leaves the surface unvaried when the wheel detaches: thus, the actual depth of cut must be "clipped" to zero if negative:

$$
\begin{aligned}
& I(\theta)=y_{N L}\left(X(\theta)+K_{1} r(\theta-\alpha)-K_{2} r(\theta-\right. \\
& (\pi-\beta))-r(\theta-2 \pi))
\end{aligned}
$$

where:

- $\quad K_{1}$ and $K_{2}$ are the well-known coefficients relating WP displacement at the grinding contact to radius variation at work rest and rubbing wheel contacts respectively: $K_{1} \triangleq \sin \beta / \sin (\alpha+\beta) \quad$ and $K_{2} \triangleq$ $\sin \alpha / \sin (\alpha+\beta)$. The contact angles depend on 
work-height $h_{w}$ and work-rest angle $\gamma$ where $\alpha=\pi / 2-\gamma-\beta$ and $\beta=\beta_{s}+\beta_{c}$ (see Fig. 1). Angles $\beta_{s}$ and $\beta_{c}$ are given by $\beta_{s}=\sin ^{-1}\left(2 \cdot h_{w} /\left(d_{s}+d_{w}\right)\right) \quad$ and $\quad \beta_{c}=$ $\sin ^{-1}\left(2 \cdot h_{w} /\left(d_{c w}+d_{w}\right)\right)$. By introducing $v \triangleq \beta / \beta_{s}$, the grinding set-up is completely defined by the 3-uple $\{\gamma, \beta, v\}$.

- $y_{N L}(\cdot)$ is a two-segment piecewise function expressing the "clipping" nonlinearity due to wheel WP detachment:

$$
y_{N L}(u) \triangleq \begin{cases}u & \text { if } u \geq 0 \\ 0 & \text { otherwise }\end{cases}
$$

Substituting Eq.(2) into Eq.(1), it yields:

$$
\begin{aligned}
& r(\theta)=y_{N L}\left(X(\theta)+K_{1} r(\theta-\alpha)-K_{2} r(\theta-\right. \\
& (\pi-\beta))-r(\theta-2 \pi))+r(\theta-2 \pi)
\end{aligned}
$$

Knowing that $\theta=\Omega t$, the phase associated to a pulsation $\omega$ can be written as $\omega t=(\omega / \Omega) \theta=n \theta$, where $n$ is the number of oscillation cycles in a WP revolution, i.e. the number of lobes. Then, in order to study system stability, Eq.(4) can be rewritten in Fourier domain with $s=j n$ (for sake of readability, the dependency on $j n$ is omitted in the notation):

$r=y_{N L}\left(X+K_{1} r e^{-j n \propto}-K_{2} r e^{-j n(\pi-\beta)}-\right.$ $\left.r e^{-2 j n \pi}\right)+r e^{-2 j n \pi}$

The nonlinearity concerned with the clipping effect (also indicated as negative depth of cut nonlinearity) is normally considered when developing numerical simulation models ${ }^{8}$, together with other nonlinearities ascribable to contact filtering (both at wheel and rubbing wheel side). In case the wheel is always touching the $\mathrm{WP}$, the nonlinearity $y_{N L}$ can be replaced with the identity and the effect of the one turn regeneration (i.e. the delay term $e^{-2 j n \pi}$ ) is canceled out. In this case, the Eq.(5) converges to the simpler linear expression that is ubiquitously adopted in literature ${ }^{6,13}$ when tackling geometric instability:

$$
r=X+K_{1} r e^{-j n \alpha}-K_{2} r e^{-j n(\pi-\beta)}
$$

The above-mentioned assumption holds when the previous and current waviness are in phase, namely, i.e. when $n$ is an integer number. On its turn, the literature supports the condition of an integer $n$ by the claim that a non-integral number of waves is "less likely to build up since the waviness must be constantly removed and replaced by a succeeding wave"21.

\subsection{Wave filtering}

According to Hashimoto ${ }^{22}$, contact filtering occurring at regulating-wheel/WP contact (denoted with $\mathrm{cr}$ ) and at wheel/WP contact (denoted with $c s$ ) can be represented by the following expression:

$$
Z_{c s / c r}(n)=\frac{1}{2}\left(1+\cos \left(\frac{l_{c s / c r}}{d_{w}} n\right)\right)
$$

where $l_{c s / c r}$ is the contact length at the interface, $d_{w}$ is the WP diameter. $Z_{c s / c r}(n)$ is the resulting low pass filter acting on the overall WP radius reduction at wheel or rubbing-wheel contact. Since the effect of both contact filtering is the same, i.e. to smooth high order lobes hindering their growth, for sake of simplicity the sole wheel/WP contact filtering is considered here. The contact length is expressed as $l_{c s} \approx \sqrt{I \cdot d_{e}}$, where $d_{e}$ is the equivalent diameter $d_{e} \triangleq\left(d_{w}^{-1}+d_{s}^{-1}\right)^{-1}$ and $I$ is the wheel-WP depth of cut.

Then, the process can be conveniently represented by the block diagram in Fig. 2, where the regeneration loops associated to lobes creation, clipping nonlinearity and contact filtering are pointed out.

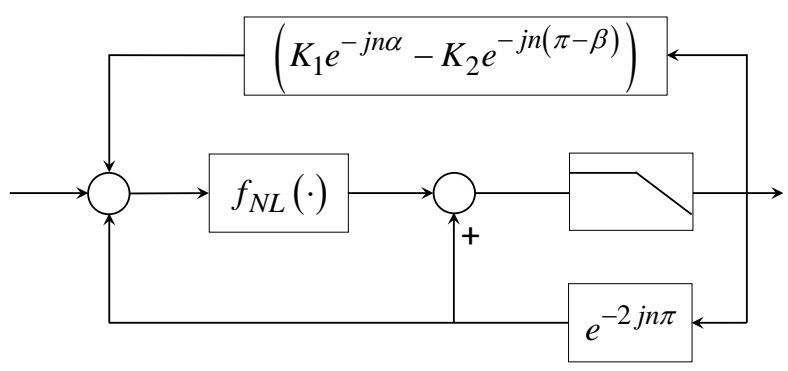

Fig. 2. Block diagram of geometric rounding mechanism

\subsection{Model of clipping nonlinearity}

To investigate clipping effect on geometrical waviness, it is useful to preserve the possibility of analyzing stability in the frequency domain. In a non-linear system, linearization must take into account the expected system behavior: in particular, it is envisaged that signal $I$, i.e. the nominal (positive or negative) wheel/WP intersection, can be depicted as a slowly varying constant plus a harmonic oscillatory term. Under those assumptions, a quasi-linear approximation can be obtained by the Describing Function (DF) approach, which is a widely known technique to study frequency response and limit cycles of nonlinear systems ${ }^{23}$. The resulting transfer functions, called describing function, depends on frequency, as in linear systems, but also on input amplitude. Given the asymmetric nature of the clipping function, a dual inputs DF has been selected (DIDF). Input and output signals are therefore described as a single harmonic term plus a bias:

$$
S(\vartheta) \approx B+A \sin 2 \pi n \vartheta
$$

$A$ is assumed to be a positive number, while $B$ can also be negative. The DFs, linking the input and output signals, are defined for $B_{\text {in }} / A_{\text {in }}$ values for which partial clipping occurs, i.e. when $A_{\text {in }}>\left|B_{\text {in }}\right|$ (elaborated from Gelb et al. $^{23}$ ):

$A_{\text {out }}=0.5 \cdot A_{\text {in }}+\frac{1}{\pi} \sin ^{-1}\left(\frac{B_{\text {in }}}{A_{\text {in }}}\right) \cdot A_{\text {in }}+$ 
$\frac{1}{\pi} \sqrt{1-\left(\frac{B_{\text {in }}}{A_{\text {in }}}\right)^{2}} \cdot B_{\text {in }}$

$B_{\text {out }}=0.5 \cdot B_{\text {in }}+\frac{1}{\pi} \sin ^{-1}\left(\frac{B_{\text {in }}}{A_{\text {in }}}\right) \cdot B_{\text {in }}+$

$\frac{1}{\pi} \sqrt{1-\left(\frac{B_{\text {in }}}{A_{\text {in }}}\right)^{2}} \cdot A_{\text {in }}$

They provide a linear relationship around a given $B_{\text {in }} / A_{\text {in }}$ value. The ratio $\mathrm{N}_{\mathrm{A}} \equiv A_{\text {out }} / A_{\text {in }}$, for a given $B_{i n} / A_{\text {in }}$, is depicted in Fig. 3. It must be noted that coupling terms appear clipping of the sinusoidal function depends on the input bias and, additionally, contributes to the average output value, given by $B_{\text {out }}$.

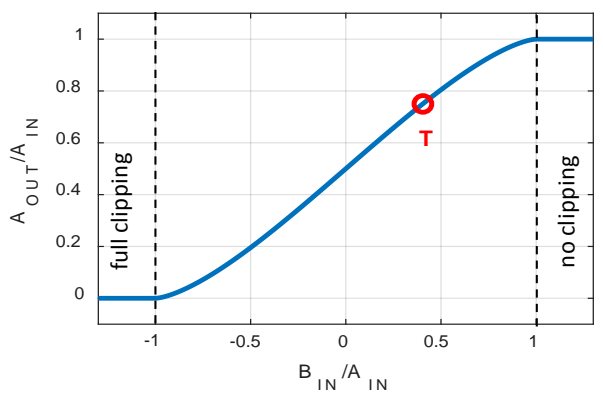

Fig. 3. I/O gain of the sinusoidal term, $N_{A} \equiv A_{\text {OUT }} / A_{I N}$

The approximation introduced by the DF approach is strictly related to difference between the adopted signal structure, constituted by a bias plus a single harmonic term, and the actual input signals. Even when autonomous oscillations in an isolated system are studied, as in our case, additional harmonics can arise: a sinusoidal input to a non-linear element produces a distorted output, with several harmonic terms. In order to get an effective application of the DF approach, the additional harmonics must be attenuated by a filtering effect of the linear elements in the feedback loop (Fig. 2 ). In the analyzed system, this effect is produced by contact filtering: its effectiveness is investigated by numerical simulations in Section 4.

The final block diagram of the process is depicted in Fig. 4, where $N_{A} \in[0,1]$ and $N_{B} \in[-1,1]$, as demonstrated above. When clipping does not occur, then $N_{A}=N_{B}=1$ and the "one-per-turn" delay loop is canceled. The dynamics is therefore defined by the delays related to WP contacts at the support and rubbing wheel. On the contrary, for a severe clipping, $N_{A}, N_{B}<<1$ : the system dynamics is dominated by the one-per-turn delay, which results in an integer number on lobes. In the next paragraph, the stability analysis will demonstrate that even a $N_{A}$ slightly lower than 1 is sufficient to move the stable condition towards an integer number of lobes.

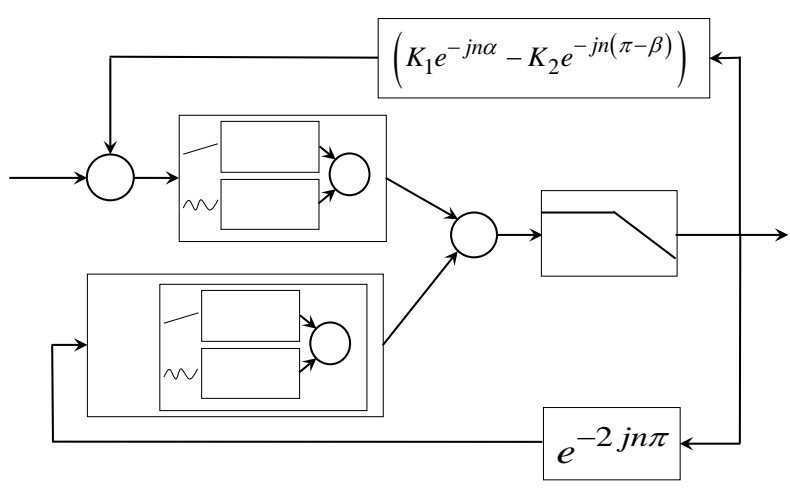

Fig. 4. Rearranged block diagram of geometric rounding mechanism with DIDF

It is worthwhile to note that the one-per-turn regeneration loop emerges also considering other phenomena at wheel/WP contact point. In particular, both dynamic and static compliance, implying an effect that is proportional to the positive actual depth of cut, yield a similar model structure ${ }^{1,24}$.

\section{Stability analysis}

System nonlinearity can produce limit cycles: for a model including a DIDF (Fig. 4), this means that, after an initial unstable divergence, $A_{I N}$ and $B_{I N}$ reach a defined steady state value. This boundary condition can be identified applying the Nyquist criterion: at frequency (i.e. lobe number) 0 for $B_{I N}$ and at lobe number $n$ for $A_{I N}$.

The loop transfer function of the system in Fig. 2, assuming the usual negative feedback, is:

$$
\begin{aligned}
& L(j n)=Z_{c S}(n)\left[N _ { N L } ( A _ { I N } , B _ { I N } ) \left(K_{2} e^{-j n(\pi-\beta)}-\right.\right. \\
& \left.\left.K_{1} e^{-j n \alpha}+e^{-j 2 \pi n}\right)-e^{-j 2 \pi n}\right]
\end{aligned}
$$

where $N_{N L}$ represents the gain of the clipping element linearized by the DF approach, to be replaced by $N_{A}$ or $N_{B}$ in the corresponding analyses. Firstly, the steady state condition for the bias terms in the loop is analyzed. The infeed input $X$ grows linearly with WP rotation, by a quantity of $U$ per turn (infeed per turn), i.e., in the frequency domain:

$$
X(j n)=\frac{U}{j 2 \pi n}
$$

The gain of the one per turn regenerative loop goes to infinity as $n \rightarrow 0$ and can be approximated as:

$$
\frac{B_{r}}{B_{\text {OUT }}}=\frac{1}{1-e^{-j 2 \pi n}} \underset{n \rightarrow 0}{\longrightarrow}-j \frac{1}{2 \pi n}
$$

The steady state condition is written cutting the loop at $B_{\text {OUT }}$, taking into account the loop gain for $n=0$ (in particular, is assumed $Z_{c s}(0)=1$ ), exploiting Eq.(12) and Eq.(13): 


$$
\begin{array}{r}
{\left[B_{\text {OUT }}\left(\frac{-j}{2 \pi n}\right)\left(K_{1}-K_{2}-1\right)+\frac{U}{j 2 \pi n}\right]} \\
\cdot N_{B}\left(A_{I N}, B_{I N}\right)=B_{\text {OUT }} \\
\Rightarrow \bar{B}_{\text {OUT }}=\frac{U}{1+K_{2}-K_{1}}
\end{array}
$$

that expresses the classic link between infeed motion and theoretical material removal in centerless grinding. The prescribed $\bar{B}_{\text {OUT }}$ establish, via the $N_{B} \mathrm{DF}$, an implicit constraint between $B_{I N}$ and $A_{I N}$ :

$$
\begin{aligned}
& \bar{B}_{\text {OUT }}=\frac{U}{1+K_{2}-K_{1}}=0.5 \cdot B_{I N}+\frac{1}{\pi} \sin ^{-1}\left(\frac{B_{I N}}{A_{I N}}\right) . \\
& B_{I N}+\frac{1}{\pi} \sqrt{1-\left(\frac{B_{I N}}{A_{I N}}\right)^{2}} \cdot A_{I N}
\end{aligned}
$$

The corresponding $B_{I N}=f\left(A_{I N}\right)$ relation is plotted in Fig. 5 (a), together with bisectors $B_{I N}= \pm A_{I N}$ : the analysis starts when clipping occurs (i.e. $A_{I N}>B_{I N}$ ). For

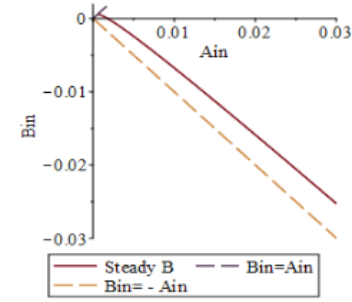

(a)

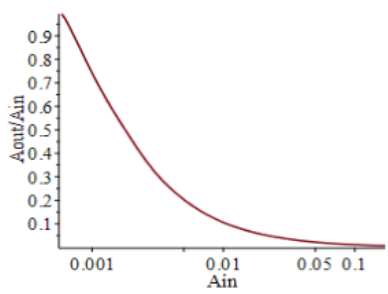

(b)
Fig. 5. (a): BIN, AIN relationship that satisfy B steady state condition. (b): corresponding gain for the harmonic term

very large values of $A_{I N}, B_{I N}$ becomes largely negative: most of the harmonic signal is clipped, while the remaining positive part produces a positive average actual material removal in output, $B_{\text {OUT }}$, as required by the steady state condition.

Now, let a sample grinding operation be considered, which will be used to illustrate the various results throughout the article: $\mathrm{U}=0.001 \mathrm{~mm}$ (corresponding to a depth of cut of $\mathrm{I}=0.00056)$, characteristic angle parameters tuple $\left\{20^{\circ}, 10^{\circ}, 0.38\right\}, d_{w}=50 \mathrm{~mm}$ and $d_{s}=300 \mathrm{~mm}$.

When $B_{\text {IN }}$ and $A_{\text {IN }}$ fulfill the stability constraint, the amplification of the harmonic term, $A_{\text {OUT }} / A_{I N}$, depicted in Fig. 5 (b), goes from 1 to 0 . This information is used while analyzing the stability condition for the harmonic term, here below. In this case the steady state condition is defined by the amplitude $A$ and "frequency" $n$. The limit case is found applying the usual Nyquist criterion on the characteristic equation:

$$
Q(j n) \triangleq L(j n)+1
$$

In a system with a DF, it's useful to solve $Q(j n)$ for $N_{A}\left(A_{I N}, B_{I N}\right)$, to identify gain values and corresponding wave numbers for which the limit stability condition can be reached:

$$
\begin{aligned}
& N_{A}\left(A_{I N}, B_{I N}\right) \\
& =\frac{Z_{c S}^{-1}(n)-e^{-j 2 \pi n}}{-K_{2} e^{-j n(\pi-\beta)}+K_{1} e^{-j n \alpha}-e^{-j 2 \pi n}}
\end{aligned}
$$

The complex function at the right-hand side of the previous equation is plotted in Fig. 6. Possible steady state solutions correspond to intersections with the real axis between 0 and 1 , which is the range covered by $N_{A}\left(A_{I N}, B_{I N}\right)$ (see Fig. 5 (b)).

According to Rowe ${ }^{15}$, let the geometric stability parameters be defined: $P_{A} \triangleq \mathfrak{N}(Q(j n))$ and $P_{B} \triangleq$ $\mathfrak{I}(Q(j n))$. Whereas the system becomes stable when $P_{A}$ becomes negative, this quantity can be approximately interpreted as a measure of lobes growth rate. Assuming an increasing level of clipping with a growing lobes amplitude, $N_{A}$ (where $A_{I N}, B_{I N}$ are understood for ease of notation) decreases and the situation evolves as illustrated in Fig. 7 for the considered example. When the depth of cut is always positive and the system is linear $\left(N_{A}=1\right)$, the minimum level of geometric stability parameter falls at 5.3 lobes, that is a not-integer number of lobes and does not correspond to the experimental evidence for the final WP geometry. When $N_{A}$ decreases, the stability parameter associated to noninteger lobes increase, while the one associated to integer lobes remains almost constant, thus, becoming the most critical: in our case, this happens at $n=15.96$ (Fig. 6). The less severe nonlinearity considered in the example, $N_{A}=0.75$, corresponds to a ratio between the nominal infeed and waviness amplitude (i.e., $\mathrm{B}_{\mathrm{IN}} / \mathrm{A}_{\mathrm{IN}}$ ) of 0.41 (point " $T$ " in Fig. 3), that is greater than in common practice. Lobes growth rate $\hat{\sigma}$ and the number of lobes $\hat{n}$ can be precisely identified by imposing the Nyquist stability limit on a translated D-contour, that is:

$Q(\sigma+j n)=0 \stackrel{\text { yields }}{\longrightarrow}(\widehat{\sigma}, \hat{n})$

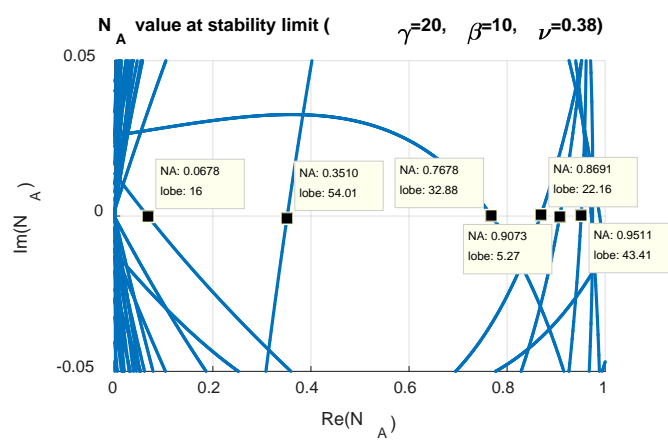

Fig. 6. NA at stability limit for the considered example 


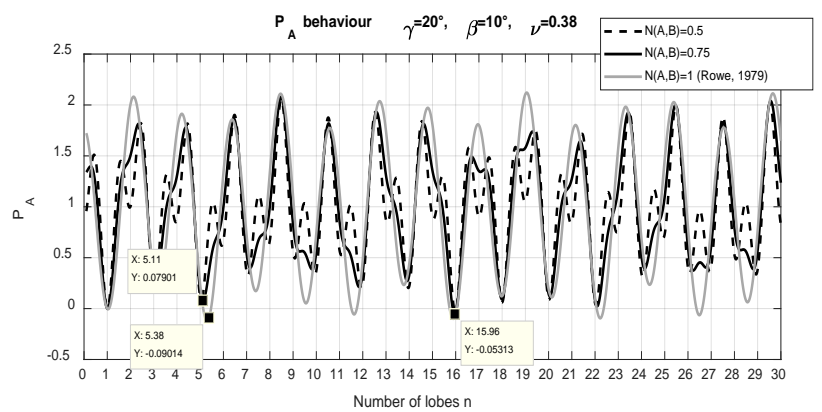

Fig. 7. Geometric stability parameter for $\gamma=20^{\circ}, \beta=10^{\circ}$ and $v=0.38$

The $\hat{\sigma}$ values for $N_{A}=1$ and $N_{A}=0.75$ have been computed. As the clipping effect reduces the theoretical material removal, the lobes growth rate associated to the initial linear step is higher than the growth rate when the nonlinearity is significantly developed, passing from 0.042 to 0.013 . The Nyquist diagram for $N_{A}=0.75$ is plotted in Fig. 8

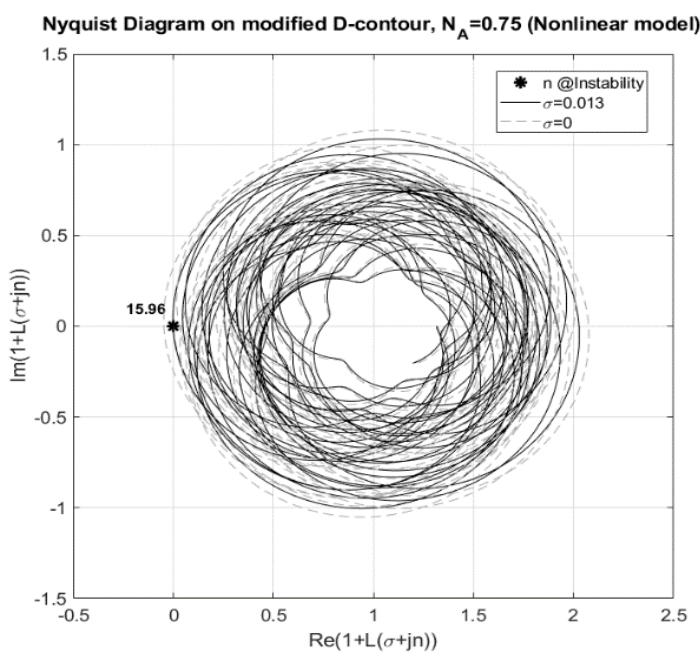

Fig. 8. Nyquist diagram with modified $D$-contour for $N A=0.75$ (for the example for $\gamma=20^{\circ}, \beta=10^{\circ}$ and $v=0.38$ )

The nonlinear model can be exploited to compute the regions of geometrical instability proposed by Rowe ${ }^{25}$. In our case, the maximum values of $P_{A}$ parameter is not computed considering only integers lobes number, but even fractional values. It can be observed that Rowe's result (Fig. 9-a) is very similar to the same diagram obtained through the present approach (Fig. 9-b); the main difference concerns the lobes number, which is now fractional (Fig. 9-c).

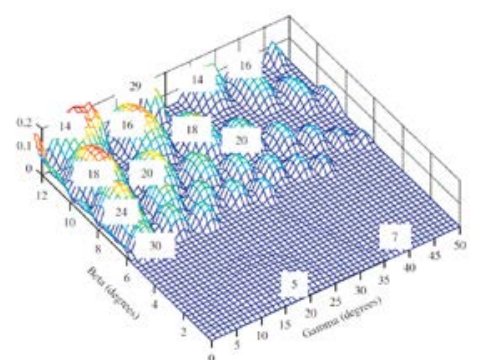

(a)

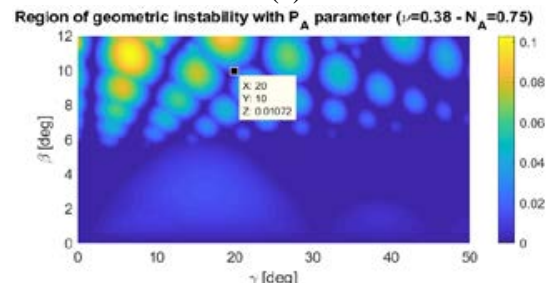

(b)

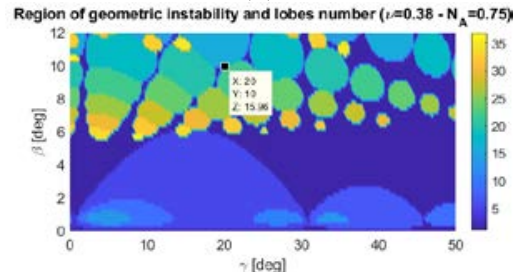

(c)

Fig. 9. Regions of geometric instability measured by PA parameter: a) imposing an integer number of lobes (Rowe 25); b) by the proposed approach, with free lobe number: c) resulting number of lobes. The noted point is the examined example.

\section{Numeric simulation results}

In order to study the lobes evolution in time-domain (more precisely, in "revolution" domain), a numeric simulation model has been developed and applied to the sample grinding process. The WP is represented by a circular array of 72000 elements, representing the WP radius reduction at a given angular position. The contact filtering illustrated in Section 2.2 has been implemented by a 0 -phase symmetric FIR filter of order 643: such a high order is necessary to make the filter fit properly the $Z_{c s}(n)$ of Eq.(7) for the contact length of corresponding to the given example, i.e. $l_{c s} \approx 0.16$. The initial WP shape is perfectly circular, namely, $r(\theta)=0 \forall \theta$ : the Fig. 10. The initial number of lobes of 5.3 (fractional) changes in 15.96 (almost integer) as the process evolves and clipping takes place. A similar result is pointed out by the time-frequency analysis of Fig. 11: the growth of 5.3 lobes is rather rapid, while dropping down as soon as clipping occurs. Moreover, it can be noted that, as the contact filter $Z_{c s}$ bandwidth is near 180 lobes, the 54 lobes component is able to slowly grow after the 16 lobes component has reached the steady state. In general, in case of long processing time, the achievement of a limit cycle for the lobes component initially more critical could give way to another component. Even though the DIDF approach developed herein is useful to predict the first and often more critical lobes number, it fails when more than one harmonic are simultaneously significant, 
as a base assumption of the DF approach is violated.
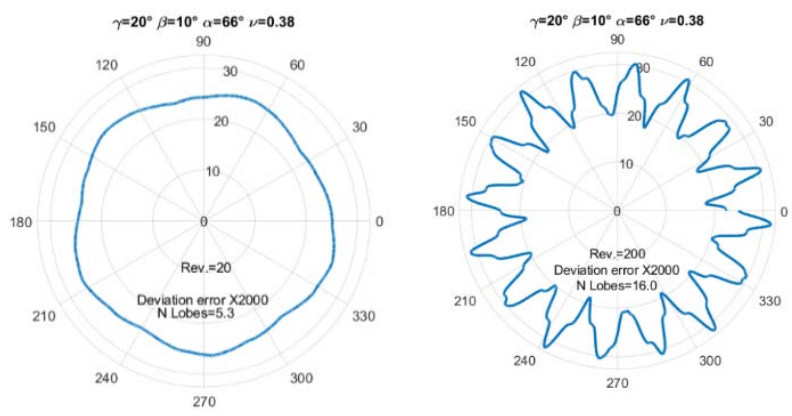

Fig. 10. WP geometry after 20 and 200 revolutions

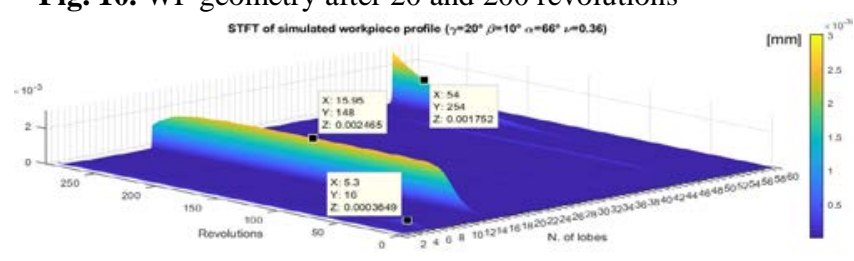

Fig. 11. Time-frequency analysis of WP profile

\section{Conclusions}

In this work we demonstrated that, dealing with pure geometrical instability, the formation of an integer number of lobes can be ascribed to clipping nonlinearity, occurring when the theoretical wheel/WP intersection is negative, without invoking other additional assumptions. The nonlinearity is modeled by a quasi-linear approximation based on the Describing Function (DF) approach. The stability analysis carried out by the Nyquist criterion yields a number of lobes coherent with the results presented in the literature. More precisely, it yields that, when clipping occurs, the most instable harmonic has the same quasi-integer number of lobes, while the usual linear model (without clipping) produces a different and non-integer number of lobes. Even though the nonlinear behavior of the system results in a limit-cycle, we highlighted that the evolution towards the steady state (final waviness amplitude) is very slow and rarely attained for typical industrial grinding operations. Moreover, in the considered example, which is on the boundary between regions characterized by different number of lobes, the evolution of the waviness entails the contemporary presence of more than one harmonic component. In this case, the assumptions of DIDFs are not satisfied and the steady state solution must be tackled by a different approach (for instance, the harmonic balance method).

Future activities will be aimed at verifying the aforementioned results by means of an experimental campaign on a commercial grinding with low-carbon steel WPs. In particular, experiments will demonstrate that, for a severely unstable grinding operation, clipping phenomenon occurs, strongly affecting lobes growth rate. Machine and process stiffness will be taken into account. Firstly, the stiffness factor will be identified by means of exponential decay method during spark-out. Thereafter, a sequence of grinding tests characterized by an increasing duration will show that the lobes growth rate is not constant and incompatible with the sole stiffness factor damping effect. In case of steady states with a dominant harmonic component, the final WP waviness amplitude will be compared with the predicted one. This latter information could be extremely useful for the optimal setup of a centerless grinding operation.

\section{References}

1. S.S. Zhou, J.R. Gartner \& T.D. Howes, CIRP Ann. Manuf. Technol. 45(1), 341-346 (1996)

2. W.B. Rowe, Principles of Modern Grinding Technology (Second Edition) (Elsevier Inc, 2014)

3. I. Gallego, CIRP Ann. - Manuf. Technol. 56(1), 347-352 (2007)

4. F.I. Klocke, D. Friedrich \& B. Linke, CIRP Ann. 53(1), 275-280 (2004)

5. A. Dall, Mech. Eng. ASME 58, 325-329 (1946)

6. S. Yonetsu, Proc. Fujihara Meml. Fac. Eng. Keio Univ. 12, 184-202 (1959)

7. W.B. Rowe \& M. Barash,. Int. J. Mach. Tool Des. Res. 4(2), 91-116 (1964)

8. W.B. Rowe, M.M. Barash \& F. Koenigsberger, Int. J. Mach. Tool Des. Res. 5(4), 203-215 (1965)

9. W.B. Rowe \& F. Koenigsberger, Int. J. Mach. Tool Des. Res. 4(3), 175-187 (1965)

10. D. Barrenetxea, et al. Int. J. Mach. Tools Manuf. 84, 17-32 (2014)

11. I.D. Marinescu, M.P. Hitchiner, E. Uhlmann, W.B. Rowe \& I. Inasaki, Handbook of Machining with Grinding Wheels (2006) ISBN 1-57444-671-1

12. J.G. Giménez \& F.J. Nieto, Int. J. Mach. Tools Manuf. 35(9), 1291-1307 (1995)

13. R. Bueno, M. Zatarain, J.M. Agunagalde \& F. Le Maitre, CIRP Ann. - Manuf. Technol. 39(1), 395-398 (1990)

14. M. Miyashita, F. Hashimoto, A. Kanai \& K. Okamura, CIRP Ann. - Manuf. Technol. 31(1), 221-223 (1982)

15. W.B. Rowe \& D.L. Richards, J. Mech. Eng. Sci. 14(2), 155-160 (2016)

16. W.B. Rowe, M. Miyashita \& W. Koenig, CIRP Ann. - Manuf. Technol. 38(2), 617-625 (1989)

17. I. Gallego, R. Lizarralde, D. Barrenetxea, P.J. Arrazola \& S. Sebastian, Precision, Stability and Productivity Increase in Throughfeed Centerless Grinding. CIRP Ann. 55(1), 4-7 (2006)

18. R. Lizarralde, D. Barrenetxea, I. Gallego, J.I. Marquinez \& R. Bueno, Practical application of new simulation methods for the elimination of geometric instabilities in centerless grinding. CIRP Ann. - Manuf. Technol. 54(1), 273-276 (2005)

19. F. Hashimoto \& G.D. Lahoti, CIRP Ann. - Manuf. Technol. 53(1), 271-274 (2004) 
20. J. Madariaga et al., J. Manuf. Sci. Eng. 132(1), 011014 (2010)

21. W.B. Rowe, Precis. Eng. 1(2), 75-84 (1979)

22. F. Hashimoto \& S. Zhou, CIRP Ann. Technol. 49(1), 225-230 (2000)

23. A. Gelb \& W.E. Vander Velde, Multiple-Input Describing Functions and Nonlinear System Design (McGraw-Hill, 1968)

24. F. Hashimoto et al., CIRP Ann. - Manuf. Technol. 61(2), 747-770 (2012)

25. W.B. Rowe, Int. J. Mach. Tools Manuf. 82-83, 1-10 (2014) 